\title{
A clinical longitudinal study 323 porcelain laminate veneers. Period of study from 3 to 11 years
}

\author{
Maria Granell-Ruiz ' , Antonio Fons-Font ${ }^{2}$, Labaig-Rueda C ${ }^{2}$, Martínez-González A ${ }^{3}$, Juan-Luis Román- \\ Rodríguez $^{4}, M^{\mathrm{a}}$ Fernanda Solá-Ruiz ${ }^{1}$
}

\begin{abstract}
${ }^{1}$ Associate Professor U.D. Dental Prosthesis and Occlusion. Department of Stomatology. University of Valencia
${ }^{2}$ Full Professor U.D. Dental Prosthesis and Occlusion. Department of Stomatology. University of Valencia

${ }^{3}$ Assistant Professor in charge of Prosthesis II. Faculty of Dentistry. University Cardenal Herrera - CEU. Valencia

${ }^{4}$ Adjunct Professor U.D. Dental Prosthesis and Occlusion. Department of Stomatology. University of Valencia
\end{abstract}

Correspondence:

Unidad de Prostodoncia y Oclusión

Edificio Clínica Odontológica

C/Gascó Oliag $n^{\circ} 1$

46010 Valencia Spain

maria.granell@uv.es

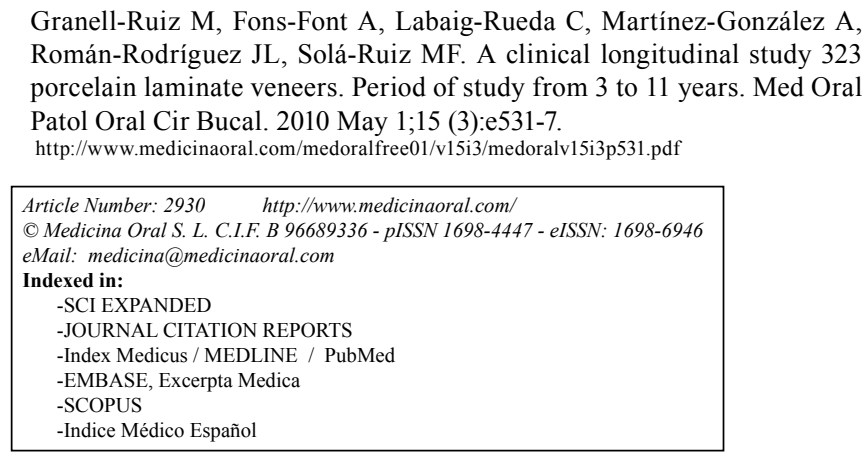

\begin{abstract}
Objective: The objective of this study was to evaluate the clinical results of 323 porcelain laminate veneers over a period of 3 to 11 years.

Material and Methods: This study included 70 patients, aged between 18 and 74 years. Of the 323 total veneers, 124 were of a simple design and 199 were of a functional design. The condition of the soft tissues and hygiene, in addition to the condition of the abutment teeth, the restorations and patient satisfaction were all clinically examined. In carrying out the statistical analysis, a descriptive approach was taken in analyzing the data. The Kaplan Meier method was used for statistically analyzing the survival rates of the porcelain laminate veneers.

Results: Analysis of the soft tissue revealed marginal recession in $7.7 \%$ of the cases, and in $21.6 \%$ of the cases, bleeding was detected on probing. Analysis of the condition of the abutment teeth revealed secondary caries and hypersensitivity in $3.1 \%$ of the cases, and changes in pulp vitality were observed in $2.8 \%$ of the cases. In studying the condition of the restorations, marginal integrity was observed to be excellent in $98 \%$ of the cases, slight marginal pigmentation was present in $39.3 \%$ of the cases, fractures were present in $4 \%$ of the cases and decementation in $9 \%$ of the cases. The degree of patient satisfaction was considered to be excellent in $97.1 \%$ of the cases.

Conclusions: Porcelain laminate veneers are a predicable treatment option that offer excellent results.
\end{abstract}

Key words: Clinical evaluation, veneer, marginal adjustment, fracture, porcelain laminate veneer. 


\section{Introduction}

Due to their high esthetic appeal, as well as their proven biocompatibility and long-term predictability, porcelain laminate veneers have become a routine restorative procedure for treatment of teeth in the front area of the mouth (1). They respresent a therapeutical alternative for a large number of patients, generally intended to satisfy the esthetic needs of the population, and in other situations, they are used in conjunction with other types of therapy.

Currently, the use of porcelain laminate veneers is almost routine for patients with alterations in the shape and color of their teeth, as well as in slight malpositions, closing of gaps, etc., a set of indications that are modified and broadened as dentists acquire more confidence in the technique and as the ceramics improve in their esthetic and resistence properties (2,3). (Fig. 1 and 2) As with any technique in the process of development, the use of porcelain laminate veneers requires both

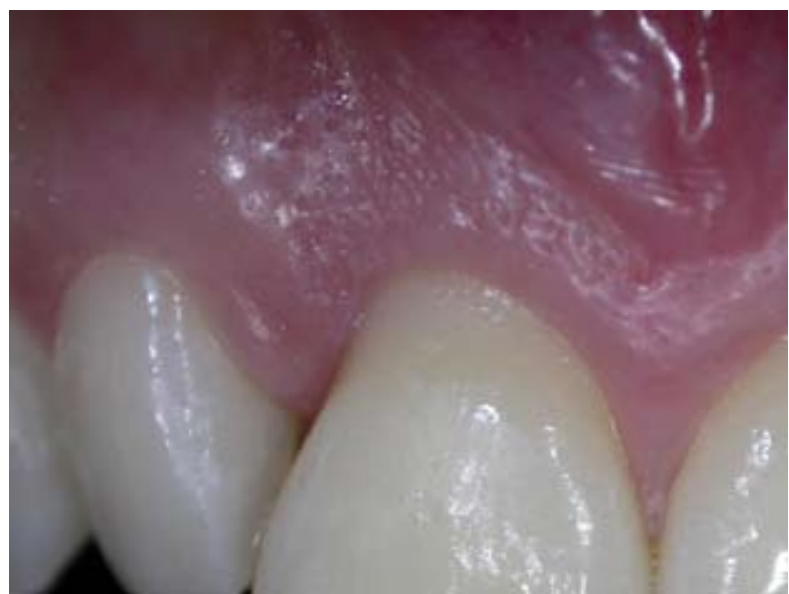

Fig. 1. Image prior to treatment with four porcelain laminate veneers.

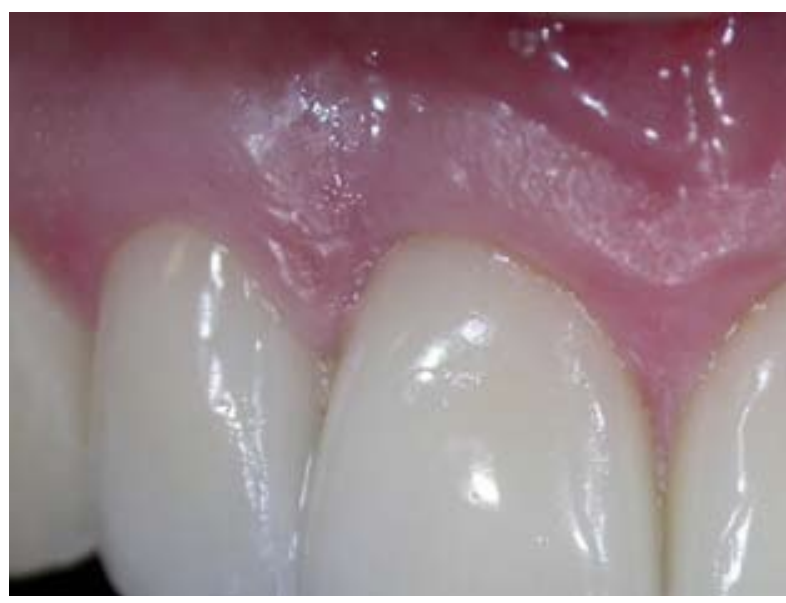

Fig. 2. Case resolved with four porcelain laminate veneers. mid-term and long-term studies in order to verify their usefulness and allow them to continue to be used, establishing, reducing, or expanding their indications for the population (3-12).

These techniques have been used at the Prosthodontics and Occlusion Teaching Unit of the Department of Medicine and Dentistry of the University of Valencia since 1985, which has now treated a significant number of patients with porcelain laminate veneers in response to their esthetic demands.

In this study, we propose to review a sample of those patients, which should serve to demonstrate how the passing of time affects these treatments in terms of their esthetical, biological and functional aspects.

\section{Material and Methods}

Between the years 1995 and 2003, we placed 323 porcelain laminate veneers, all of which were made with IPSEmpress ceramic (Ivoclar ${ }^{\circledR}$, Schaan, Lichtenstein) for the purpose of standardizing the results and eliminating possible variables that could originate from the use of different ceramics.

The study population included a sample of 70 patients (53 women and $17 \mathrm{men}$ ). We observed that the majority of the patients were between the ages of 31 to 40 years old. There were only three patients treated who were under the age of 20 , and five patients over the age of 60. The average age of the patients examined was 46 years old, with the range of ages between 18 and 74 years old.

Of the total 323 veneers, 124 were of a simple design, covering only the vestibular surface of the tooth, and 199 corresponded to what we called "functional" restorations, covering the incisal edge and part of the palatal/ lingual side of the tooth with $1 \mathrm{~mm}$ height palatal chamfer. The majority, 238 of the veneers were located in the upper arcade, whereas 85 were located in the lower arcade. Of the restorations made in the upper arcade, 97 were made in the central incisors, 82 in the lateral incisors, 49 in the canine teeth, and 10 in the premolars. As for the lower arcade, 31 of the veneers were located in the central incisors, 31 in the lateral incisors, 19 in the canine teeth, and 4 in the premolars (Table 1). We observed that the tooth that is most often restored is the central incisor, with a decrease in the frequency of restorations of other teeth as we move towards the back of the mouth.

All of the patients were treated at the Prosthodontics and Occlusion Teaching Unit of the University of Valencia School of Medicine and Dentistry by a team that had followed the same method when placing the veneers. Preparation of the tooth

The shaping was carried out with conventional burs, marking a finish line in a curved bevel.

The preparation of the teeth for the porcelain laminate 
Table 1. The location of the restorations according the individual teeth is expressed.

\begin{tabular}{|l|c|c|c|c|c|}
\hline & I. Central & I. Lateral & Canine & Premolar & $\begin{array}{c}\text { Total } \\
\text { Porcelain } \\
\text { Laminate } \\
\text { Veneers }\end{array}$ \\
\hline $\begin{array}{c}\text { Upper } \\
\text { Arcade }\end{array}$ & 97 & 82 & 49 & 10 & 238 \\
\hline $\begin{array}{c}\text { Lower } \\
\text { Arcade }\end{array}$ & 31 & 31 & 19 & 4 & 85 \\
\hline $\begin{array}{c}\text { Total } \\
\text { Porcelain } \\
\text { Laminate } \\
\text { Veneers }\end{array}$ & 128 & 113 & 68 & 14 & 323 \\
\hline
\end{tabular}

veneers was, as is always the case in prosthodontics, as conservative as possible, adhering to the principle of preserving the dental tissue. More specifically, this type of treatment is characterized by a particularly conservative reduction of the tooth, approximately $0.3-0.5 \mathrm{~mm}$ in thickness on the vestibular surface of the tooth to be treated, being very careful to avoid going down to the dental tissue (simple design). The tooth preparation was most aggressive, up to $1 \mathrm{~mm}$, in those teeth that presented alterations in the color (tetracycline) or malpositions. For the cases in which we were not going to lengthen the tooth with the veneer, the tooth was only reduced in terms of thickness, not in terms of height. The incisal reduction with $1 \mathrm{~mm}$ height palatal chamfer (functional design) was carried out in those cases in which the tooth required an incisal overlap. The finish line was a curved bevel and usually juxtaginival or subgingival $(0.5 \mathrm{~mm})$ in cases of intense staining. Interproximally speaking, the contact points were broken with thick grain metal strips.

Taking of impressions

We used three different impression techiques, each depending on the case:

.The Wash-Technique using silicone addition in three different viscosities as the preferred material.

.Using an individual tray and polyethers. In this technique, we used two different consistencies of polyethers. We prescribed this technique and the one above for cases in which there were multiple dental preparations.

After taking the impression, a closed-mouth bite registration of the prepared area was taken.

.Using a front partial tray with the mouth closed, a Pre-

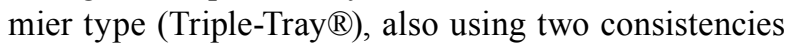
of polyethers. This technique can only be used for one or two restorations.

\section{Placement of temporary restoration}

In each case, we place temporary restorations in order to maintain the esthetics, avoid issues of sensitivity to temperature changes as well as exposure of the dentin to bacterial infection. The temporary teeth placed in the cases treated in this analysis have been performed directly in the mouth with a photopolymerizable composite mould on the carved surface, which had previously been engraved with $37 \%$ orthophosphoric acid at a central location and subsequent application of dental adhesive. This allows for adequate retention so that the composite facet does not come undone, which is also easily removable by loosening and lifting it up with a spatula.

\section{Cementation}

The cementing technique used was standardized in all the restorations included in the sample. For treating the surface of the tooth, we used a solution with a $37 \%$ concentration of orthophosphoric acid, then we applied the same dental adhesive (Syntace ${ }^{\circledR}$, Vivadent). In addition, we also proceeded to treat the internal side of our restorations using a solution containing silane and a $10 \%$ concentration fluorohydric acid. Once the two surfaces had been treated, that of the tooth and that of the restoration, we cemented the veneer, using the same cement-based composite in each case; although to avoid varying results, different colors were used depending

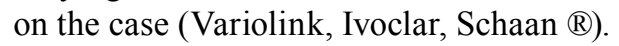

Then, where applicable, we immediately removed the coarse remains of cement bonded to the margins and the interproximal area, using a polishing bur and dental floss, respectively in each case.

Finally the occlusion was checked in order to verify that no unwanted changes were made and/or that we correctly restored the anterior guide in cases of functional veneers (2).

\section{Evaluation}

The review and collection of data established in a protocol was performed by a single browser in order to combine the criteria of the findings. All of the data was collected over the course of the year 2006. We evaluated the plaque index, gingival bleeding index, recessions, secondary caries, hypersensitivity, changes in pulp vitality, marginal pigmentation, changes in color, marginal adjustment, mechanical accidents (fissures, fractures 
and unbonding) and the degree of patient satisfaction. In carrying out the statistical analysis, a descriptive approach was taken in analyzing the data. The Kaplan Meier method (13) was used for statistically analyzing the survival rates of the porcelain laminate veneers. The survival time was defined as the period when we began to cement the restoration and ended when the veneer failed. The main criteria used in defining the failure of the veneer were the fracture of the porcelain and/or the unbonding.

\section{Results}

During the evaluation period, the results obtained were as follows:

The plaque index for the restored teeth was as follows: $71.5 \%$ showed a value of $0 ; 13.3 \%$ (43 patients) showed a value of $1 ; 10.5 \%$ (34 patients) showed a value of 2 ; and only $4.6 \%$ showed a value of 3 .

The gingival bleeding index for the restored teeth presented the following results: $78.3 \%$ (253 patients) showed a value of $0 ; 16 \%$ (52 patients) showed a value of $1 ; 5.6 \%$ (18 patients) showed a value of 2 ; and none of the teeth showed a value of 3 .

In $92.3 \%$ of the cases, there was no indication of recession, whereas $7.7 \%$ of the cases did show evidence of recession. In $6.8 \%$ of the cases, the recession was $1 \mathrm{~mm}$; in $0,6 \%$ of the cases, the recession was $2 \mathrm{~mm}$; and in only $0.3 \%$ of the cases, the recession was $3 \mathrm{~mm}$.

Upon analyzing the presence of secondary caries after the treatment, we observed that in ten of the teeth restored, $3.1 \%$ ( 7 patients) had secondary caries at the time of the revision.

The results obtained upon analyzing the sensitivity of the teeth used as pillars allowed us to verify that only three patients (10 restored teeth, which correspond to $3.1 \%$ of the sample) complained of this type of symptom after the treatment.

We also verified at the time of the revision of the restored teeth that nine of them (which correspond to $2.8 \%$ of the total amount of restored teeth) had suffered a change in their pulp vitality, four of them had suffered a change in the vitality with their corresponding root canals. In the remaining 5 restored teeth, apical areas were found to be present.

Upon studying the marginal pigmentation that could be observed in the restored teeth, it was particularly remarkable that this type of staining appeared on 127 $(39.3 \%)$ of the restored teeth. The area where this pigmentation occured appeared in the tooth-restoration interface.

As far as changes in color of the restorations that may appear over time, we observed such an alteration in only two restorations $(0.6 \%)$. Both alterations were noted by the clinic and by the patient.

Upon analyzing the degree of vertical and horizon- tal adjustment that the restorations presented, we observed that of the 323 restorations that were studied, 315 $(97.5 \%)$ did not present any vertical defect; thus, axially speaking, the adjustment of the restoration on the finish line was good. Alterations were found in only 8 restorations $(2.5 \%)$, in which the alteration was about 100 microns in 5 of the restorations; that is, the alteration was not noticeable to the naked eye and could only be detected by the tip of a probe, whereas in three of the cases, the defect was more than 100 microns and was visually noticeable. As far as horizontal defects found in the restorations, 5 cases $(1.5 \%)$ of such defects were observed in the all of the restorations, whereas no such defects were observed in the remaining 318 (98.5\%) restorations. Among these five restorations, one of these had been overcontoured on the preparation margin.

Mechanical accidents: The survivial of the restorations as far as their structural integrity is concerned, is probably an extremely important to consider, both for the patients as well as the professionals when deciding whether to use these restorations. Therefore, we analyzed the survival of the restorations based on the three most important aspects: the appearance of fissures, fractures and unbonding, or the lack thereof.

Fissures: At the time of reviewing our restorations, no fissures were observed. However, this is not to say that some of the fractures found in our restorations did not begin with a fissure, which over the course of time eventually caused a fracture in the restoration.

Fractures: We observed a total of 13 fractures $(4 \%)$, which included 11 in functional design restorations (corresponding to $84.6 \%$ of the fractures) and 2 in simple design restorations (corresponding to $15.4 \%$ of the fractures) (Fig. 3).

Decementations: The total number of decemented restorations observed in this study was 29 , which represents $9 \%$ of the sample. They were distributed homogenously, given that 14 were in functional design restorations and 15 were in simple design restorations (Fig. 3).

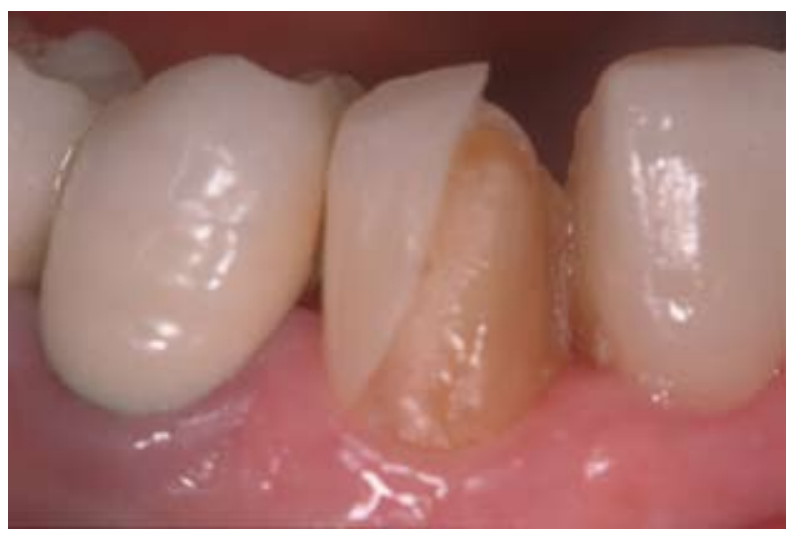

Fig. 3. Fracture of a front porcelain laminate veneer. 


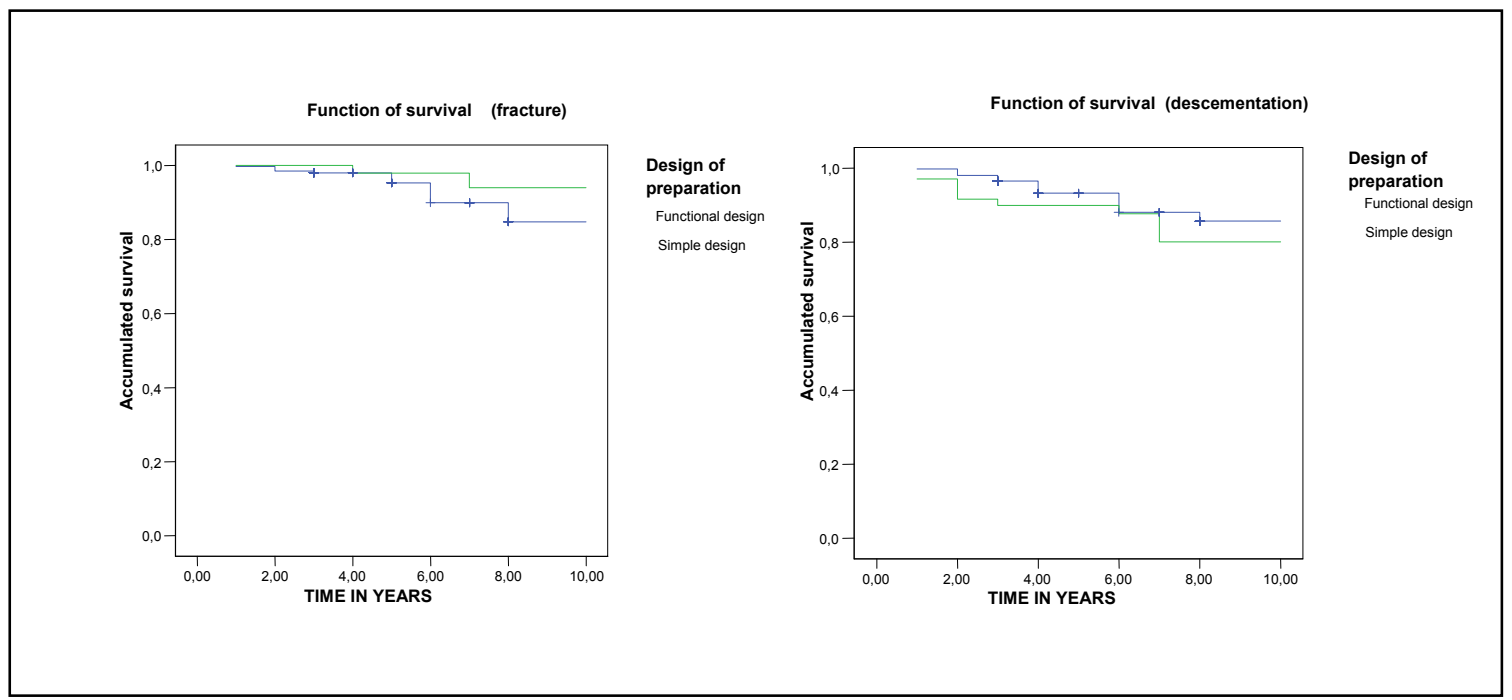

Fig. 4. Survival rate of fracture and decementation of the restoration.

According to the Kaplan-Meier method of estimation, the probability that the 323 restorations will survive fractures after 11 years is $94 \%$ in simple design restorations and $84.7 \%$ in functional design restorations. The probability that they will survive decementation after 11 years is $80 \%$ in simple design restorations and $85.6 \%$ in functional design restorations (Fig. 4).

Finally, we include the results of the survey conducted on patients regarding their opinion about the restorations, distinguishing between the esthetic result and the functional result (comfort and chewing), based on a scale of 0 to 10 . We observed that $97.1 \%$ of the patients (68 patients) were comfortable with the restorations and had no problems with chewing. As far as the esthetic result, again, 97.1\% of the patients (68 patients) commented that they were satisfied with the esthetic results of the restorations.

\section{Discussion}

There have been numerous longitudinal clinical studies available on the behavior of porcelain veneers (3-12). We were able to verify that the clinical studies are necessary in order to evaluate the behavior of the materials restored, given that there are certain intraoral conditions that cannot be reproduced in a laboratory. For this reason, in vivo studies have proven to be necessary in considering a veneer as an acceptable permanent restorative treatment. Retrospective studies may offer a reliable observation of the clinical behavior of the materials and techniques.

On the other hand, there are numerous in vitro studies on veneers (14-16). However, such studies do not offer the same prognostic value and long-term predictability of the treatment as that of in vivo studies. Due to rapid and constant changes in materials and technology, the longitudinal clinical studies that have been conducted over more than rial and techniques used. Thus, in vitro studies have a greater impact, although not as useful as in vivo studies.

Discussion of the results:

A total of $71.5 \%$ of the restored teeth were free from bacterial plaque, which is very similar to the results found in other studies that show that the teeth restored with these types of restorations have less plaque than other materials 5 years clearly contribute results that are scientifically very useful, although sometimes obsolete as far as the mate used in dental restoration, even including the dental enamel, given that plaque is easily eliminated from these types of surfaces and/or because the life of the bacterial plaque is significantly reduced on the porcelain material itself (17).

In $21.7 \%$ of the teeth restored with porcelain laminate veneers, bleeding occured on probing. These results are comparable to those obtained in other studies similar to ours (4-11).

In our study, recessions were observed in $7.7 \%$ of the teeth treated. In studies conducted by Dumfahrt et al. (11), recessions were observed in $30 \%$ of the cases, which was justified by explaining that recessions are frequent in patients with good oral hygiene and that the proportion of individuals with recessions increases with age. Given that his study was carried out over a prolonged period of time, the recessions may be considered as a consequence of the passing of time.

Secondary caries were found to be present in 10 of the restorations ( 7 patients), which is a high percentage $(3.1 \%)$ if we compare it to other studies $(5,9,18)$ where the percentage of caries was between $1 \%$ and $2 \%$. According to studies by other authors $(11,19)$, they even report that no secondary caries were found to be present 
upon reviewing their restorations. In order to be able to explain the incidence of secondary caries, we have observed that the majority of them appear in patients who present plaque indexes of 2 , in addition to rather high CAO (Caries Absent Occluded) indexes. Therefore, we believe that this may be explained by the poor hygiene of these patients and their high tendency to suffer lesions due to caries.

In our experience, there were several patients who complained of sensitivity in the teeth that were treated with this type of restoration during the first few days after it was placed, but such sensitivity seems to gradually disappear over time. However, in the review that we carried out, some of the patients still complained of sensitivity, which can be explained by the fact that the reason these patients came to our clinic was due to dental malposition, and the cutting was more agressive in these cases. Thus, we think that this may be the reason, given that none of these patients presented any alterations or filtrations. According to our review of clinical studies conducted by other authors $(2,10)$, none of their patients compained of sensitivity at the time of the revision.

As for dental vitality, we observed that nine teeth (corresponding to $2.8 \%$ of the sample) that were vital at the time of beginning the treatment, had suffered a change in their pulp vitality after a few years. Pneumans et al. (20) in one of his clinical study, also had to perform extractions of two teeth in fairly extensive composite restorations in the interproximal space. On the other hand, there are authors $(2,11)$ whose studies show no results of any tooth that suffered a loss in pulp vitality.

Our sample revealed that $39.3 \%$ of the marginal pigmentation observed was found to be slightly higher than the results of other studies $(8,20)$ in which pigmentation was produced in $22 \%$ and $25 \%$ of the restorations, and even much higher in other studies (2), in which the pigmentation only appears in $7 \%$ of the restorations.

As far as changes in the color of the restorations that may appear over time, as mentioned above in the results, we observed such an alteration in only two restorations $(0.6 \%)$. Both alterations were noted by the clinic and by the patient. Our results are very similar to those found by the majority of authors $(7,18)$.

If we consider both the vertical defects as well as the horizontal defects found in our study, our results are quite similar to those obtained in other studies $(10,19)$, in which the adjustment is excellent in $65-98 \%$ of the restorations.

\section{Analysis of mechanical accidents.}

Fissures: The fact that in our study there were no fissures or cracks in the restorations may be due to the use of high-resistance porcelain (IPS-Empress). The majority of the authors $(10,20)$ do not consider small fissures produced in their restorations to be failures.

Fractures: In our study, fractures were found in $4 \%$ of the cases, which is very similar to that found in studies conducted by other authors $(7,18)$, The majority of clinical studies compared $(10,19)$ report very low rates of fractures, between 0 and 1\%. However, other authors have reported much higher rates of fractures: Christensen et al. (5), 13\% after 3 years, and Walls et al. (8), $14 \%$ after 5 years, explaining that these results are due to the fact that the majority of these patients had a history of bruxism, and that conventional feldspatic porcelain was used, which has a lower mechanical resistance than that of high-resistance feldspatic porcelain.

In our study, we observed a higher percentage of fractures of the restorations in those patients who wore bruxismmouth guards.

In addition, we observed a higher incidence of fractures produced in fuctional design restorations (11 fractures) in comparison to simple design restorations (2 fractures). In this case, we think that the patients' history of bruxism may be responsible for the failure, as opposed to the design of the dental preparation itself. Patients with bruxism, who will receive rehabilitation of the anterior guide, are invariably treated with functional design veneers, which is why this dental preparation design is often placed in high-risk patients.

Decementations: The number of decementations found in our study was also remarkable $(9 \%)$, which generally corresponds to patients with bruxism and to teeth with large composite reconstructions and less enamel to which the restoration must be cemented. In their in vivo studies, some authors $(6,9)$ report high incidences of decementation of the restorations due to the existence of composite reconstructions on the teeth. According to other authors $(3,8)$, the decementation is not considered a failure, because the restoration can be recemented. The use of the Kaplan-Meier method (13) serves to document the rate of survival of any restoration once it is cemented in the mouth.

In our study, we observed that the rate of survival of a fracture after 11 years was $94 \%$ for simple design restorations, whereas the survival rate was $85 \%$ for functional restorations. If we compare our results with those obtained by other authors $(2,3,10,11,20)$, we observe that the survival rate we obtained is lower for functional design restorations, and the survival rate for simple design restorations is rather similar to that obtained by those authors, in which the survival rate was between $91 \%$ and $100 \%$. This relatively low survival rate for our functional design restorations in comparison with other studies $(2,3)$, may be justified due to the design of the dental preparation that we used (extension of the restoration to the back side of the tooth, leaving a fine bevel). For this reason, and due to in vitro studies (14-16), authors such as Magne et al. (2) and Fradeani et al. (3) changed the design of the functional restorations called "butt joint" (incisal reduction without a palatal chamfer). 
Other authors $(6,9)$ show less favorable results, likely related to incorrect cement application techniques.

In the majority of the clinical studies on this type of restoration, the degree of patient satisfaction has been a factor taken into account. The percentage of patients who report being completely satisfied with this type of restoration is reported to be between $80 \%$ and $100 \%$. Our study reveals results that are very similar to those found by other authors $(5,20)$.

\section{Conclusions}

1. The longitudinal clinical studies (in vivo) are necessary in order to evaluate the behavior and predictability of the materials restored, due to certain intraoral conditons that cannot be reproduced in the laboratory.

2. The design of the dental preparation consisted of a limited reduction of the vestibular surface (simple design) by $0.3-0.5 \mathrm{~mm}$. In cases where we had to lengthen the incisor edge, the preparation extended to the palatal/ lingual side of the tooth with $1 \mathrm{~mm}$ height palatal chamfer.

3. We observed fractures in $4 \%$ of the cases, with $85 \%$ of them occurring in functional design restorations, without this being statistically significant in the case of the simple design. We may conclude that it is related to bruxism, given that this design is used for high-risk patients.

4. In $9 \%$ of the cases, we observed that the bonding had come undone, generally in patients with bruxism and in teeth with large composite restorations.

5. According to the Kaplan-Meier method, the rate of survival of a fracture after 11 years was $94 \%$ for simple design restorations, whereas the survival rate was $85 \%$ rate in the case of functional design restorations.

\section{References}

1. Fons-Font A, Solá-Ruíz MF, Granell-Ruíz M, Labaig-Rueda C, Martínez-González A. Choice of ceramic for use in treatments with porcelain laminate veneers. Med Oral Patol Oral Cir Bucal. 2006;11:E297-302.

2. Magne P, Perroud R, Hodges JS, Belser UC. Clinical performance of novel-design porcelain veneers for the recovery of coronal volume and length. Int J Periodontics Restorative Dent. 2000;20:440-57.

3. Fradeani M, Redemagni M, Corrado M. Porcelain laminate veneers: 6- to 12-year clinical evaluation--a retrospective study. Int J Periodontics Restorative Dent. 2005;25:9-17.

4. Karlsson S, Landahl I, Stegersjö G, Milleding P. A clinical evaluation of ceramic laminate veneers. Int J Prosthodont. 1992;5:447-51.

5. Christensen GJ, Christensen RP. Clinical observations of porcelain veneers: a three-year report. J Esthet Dent. 1991;3:174-9.

6. Dunne SM, Millar BJ. A longitudinal study of the clinical performance of porcelain veneers. Br Dent J. 1993;175:317-21.

7. Nordbø H, Rygh-Thoresen N, Henaug T. Clinical performance of porcelain laminate veneers without incisal overlapping: 3-year results. J Dent. 1994;22:342-5.

8. Walls AW. The use of adhesively retained all-porcelain veneers during the management of fractured and worn anterior teeth: Part 2. Clinical results after 5 years of follow-up. Br Dent J. 1995;178:33740 .

9. Shaini FJ, Shortall AC, Marquis PM. Clinical performance of por- celain laminate veneers. A retrospective evaluation over a period of 6.5 years. J Oral Rehabil. 1997;24:553-9.

10. Chen JH, Shi CX, Wang M, Zhao SJ, Wang H. Clinical evaluation of 546 tetracycline-stained teeth treated with porcelain laminate veneers. J Dent. 2005;33:3-8.

11. Dumfahrt H, Schäffer H. Porcelain laminate veneers. A retrospective evaluation after 1 to 10 years of service: Part II--Clinical results. Int J Prosthodont. 2000;13:9-18.

12. Peumans M, De Munck J, Fieuws S, Lambrechts P, Vanherle G, Van Meerbeek B. A prospective ten-year clinical trial of porcelain veneers. J Adhes Dent. 2004;6:65-76.

13. Kaplan EL, Meier P. Non-parametric estimation from incomplete observation. J Am Stat Assos. 1958;53:457-65.

14. Stappert CF, Ozden U, Gerds T, Strub JR. Longevity and failure load of ceramic veneers with different preparation designs after exposure to masticatory simulation. J Prosthet Dent. 2005;94:132-9.

15. Magne P, Kwon KR, Belser UC, Hodges JS, Douglas WH. Crack propensity of porcelain laminate veneers: A simulated operatory evaluation. J Prosthet Dent. 1999;81:327-34.

16. Castelnuovo J, Tjan AH, Phillips K, Nicholls JI, Kois JC. Fracture load and mode of failure of ceramic veneers with different preparations. J Prosthet Dent. 2000;83:171-80.

17. Kourkouta S, Walsh TT, Davis LG. The effect of porcelain laminate veneers on gingival health and bacterial plaque characteristics. J Clin Periodontol. 1994;21:638-40.

18. Calamia JR. Clinical evaluation of etched porcelain veneers. Am J Dent. 1989;2:9-15.

19. Strassler HE, Nathanson D. Clinical evaluation of etched porcelain veneers over a period of 18 to 42 months. J Esthet Dent. 1989;1:21-8.

20. Peumans M, Van Meerbeek B, Lambrechts P, Vuylsteke-Wauters M, Vanherle G. Five-year clinical performance of porcelain veneers. Quintessence Int. 1998;29:211-21. 